\title{
Ventricular Arrhythmia Due to Yellow Phosphorus Poisoning
}

\section{Hiran $\mathrm{S}^{*}$}

Department of Medicine, J.L.N. Hospital and Research Centre Bhilai, India

${ }^{*}$ Corresponding author: Hiran S, Department of Medicine, J.L.N. Hospital and Research Centre Bhilai, India, E-mail: sujatahiran@gmail.com

Citation: Hiran S (2017) Ventricular Arrhythmia Due to Yellow Phosphorus Poisoning. J Case Rep Stud 5(3): 303. doi: $10.15744 / 2348-9820.5 .303$

Received Date: February 28, 2017 Accepted Date: June 27, 2017 Published Date: June 29, 2017

\begin{abstract}
Background: Ratol paste (Yellow Phosphorus) is a local and systemic toxin affecting almost all the organs of the body especially liver, kidney, brain and heart. There is no specific antidote for yellow phosphorus poisoning. Heart is one of the many organs affected by the YP. However cardiac toxicity following acute ingestion of YP has been reported rarely.

Case Report: We report a young female, who had intentionally ingested two tubes of Ratol dissolved in water having a net weight of $35 \mathrm{gm}$, containing 3\% (1.05 gm.) phosphorus. The patient presented to the hospital because of GI symptoms like nausea and vomiting. Following resolution of her symptoms in 24 hours she was discharged. She later presented in peripheral circulatory failure to the same hospital, eventually dying due to ventricular fibrillation.
\end{abstract}

Discussion: Yellow phosphorus is highly toxic compounds which are commonly used for pest control. Ratol paste, a rodenticide containing 3\% yellow phosphorus is freely available in India. It is one of the most commonly ingested rodenticides poison. Case reports of accidental and intentional ingestion has been reported due to its easy availability in India. It primarily causes hepatic toxicity leading to hepatic encephalopathy and acute hepatic failure. Cardiac toxicity includes hypotension, tachycardia, ECG changes and arrhythmia. The reported mortality after YP intoxication varies from $23 \%$ to $73 \%$, and it is directly related with the ingested dose.

Conclusion: Ventricular arrhythmia is an unusual complication of YP poisoning. It may occur as late as 48-72hours. Early improvement should not be interpreted as meaning that serious exposure has not occurred. Identifying exact component of rodenticide before discharge is mandatory. The history of poisoning should always be elicited in all cases presenting with gastrointestinal symptoms.

Keywords: Yellow Phosphorus; Ventricular Arrhythmia; Cardiac Toxicity; Ratol; Rodenticide

List of Abbreviations: Yellow Phosphorus (YP)

\section{Introduction}

Rodent control is important as rodents cause diseases and disturb the food supply. They multiply fast if left unchecked. One-dose rodenticides are often used because using a toxin that must accumulate after multiple feedings will kill only a fraction of target animals. For this purpose numerous rodenticides are available. Ratol (YP) paste and powder is easily available rodenticide in the open market in India.

YP is a commonly used rodenticide containing 3-5\%YP [1]. It is highly toxic and is responsible for many deaths after oral ingestion. Suicidal Ratol tablet overdose is the most common cause of poisoning in India [2]. Most cases of fatal or severe exposure to YP resulted in adults or children accidentally or deliberately swallowing the rat poisons [3,4] or fireworks [5]. YP is a protoplasmic poison, spontaneously combusts in air and causes cardiac, hepatic, renal, and multi organ failure when ingested. It is rapidly absorbed from the GI tract [6] and is primarily metabolized by the liver. A dose of $15 \mathrm{mg}$ of phosphorus can cause severe poisonings in humans and as little as $50 \mathrm{mg}$ may be fatal [7]. The vomitus after phosphorus ingestion is luminescent and has a characteristic garlic odour. If the patient survives the initial gastrointestinal irritation phase, secondary systemic poisoning usually in form of hepatic toxicity may ensue.

We report a case of YP poisoning who initially had not given history of oral ingestion of Ratol paste and was later readmitted in hypotension, eventually dying due to ventricular arrhythmia. This report emphasises that a history of poison ingestion should always be elicited in patients presenting with gastrointestinal manifestations.

\section{Case Report}

A 38 year old female, school teacher by profession, married with two children was admitted (First admission) with the history of vomiting and pain abdomen. The patient was given symptomatic treatment after which her symptoms abated and she was 
discharged next morning. However, she was readmitted (Second admission) a day after discharge in the early morning with the complaints of giddiness, vomiting, pain abdomen and breathing difficulty. During this admission husband disclosed the alleged history of Ratol ingestion. The patient herself admitted taking two tubes of Ratol mixed in water. The time interval between the first and second admission was 58 hours. Each tube of Ratol had a net weight of $35 \mathrm{gm}$, containing 3\% (1.05 gm) phosphorus.

On admission patient was conscious; heart rate was $148 /$ minute, BP $82 / 50 \mathrm{~mm}$ of $\mathrm{Hg}$ and respiratory rate $20 /$ minute. The extremities were cold and clammy. Systemic Examination was normal. The patient was managed by inotropes and supportive care. About six hours after admission the cardiac monitor showed ventricular fibrillation and the patient succumbed due to the cardiac toxicity of YP.

\section{Investigations}

First Admission: Hb- 9.6gm/dL, TLC-2,700/cumm, DLC - P 62\%, L 32\%, M2\% E 4\%, Platelet count- 142x10 9 /litre, Blood urea- 32 mg/dL, Serum Creatinine- 1.1mg/dL, ALT- 19 IU/ml. ALP - 194 IU/ml, serum Amylase -413 U/L, Serum Na- 142 mEq/L, K- 4.0 mEq/L.ECG-WNL, Ultrasound Abdomen- Normal.

Second Admission: Hb- 10.9gm/dL, TLC- 16,300 /cumm ,DLC -P75\%,L20\%, E 5\% Platelet 390x10 $/$ litre, Random Blood Sugar$92 \mathrm{mg} / \mathrm{dL}$, Bloodurea-44mg/dL, S.creatinine- $1.8 \mathrm{mg} / \mathrm{dl}$, Total Bilirubin- $1.7 \mathrm{mg} / \mathrm{dL}$, Direct Bilirubin- $1.2 \mathrm{mg} / \mathrm{dL}$, Indirect Bilirubin $-0.5 \mathrm{mg} / \mathrm{dL}$, Total Protein -7.2g/dL, Albumin- 4.1g/dL, A/G ratio -1.3, ALT-130IU/ml, LDH -1455 IU/L, CK 303 U/L, Serum Calcium- 7.0 mEq/L, Serum Magnesium- 1.5 mEq/L, Serum Na- 131mEq/L, Serum K- 3.6mEq/L, Serum Fibrinogen- 250 mg/dl, (150-450 mg/dL) PT- 39.0/13.0 seconds, INR- 3.34, APTT -37/30 seconds ECG- Sinus Tachycardia, ST sagging V2-V5, QTc 434 ms, P-R interval .08 seconds.

\section{Discussion}

Phosphorus is a non-metallic chemical element of the nitrogen family. The word phosphorus means light-bearer, which originates from its property of glowing when exposed to air, likely due to the formation of reactive luminescent phosphorus oxide species on its surface. It is essential for many biologic processes including synthesis of ATP. YP is used in the manufacture of rodenticides, incendiaries, phosphorus compounds, as an igniter in munitions and flares, as an igniter and pigment in fireworks, and as a semiconductor additive. Although no longer used in medicine, phosphorus is still used in some homeopathic medicines [8].YP was used in the manufacture of matches in the past and was responsible for both chronic poisoning in worker and acute poisoning from ingestion of matches.

The fate of YP following exposure by any route is an open question. However, absorption of YP by oral, dermal and inhalation routes is likely since it is highly lipid soluble. The observation of toxic effects in humans following exposure to YP suggests that YP and/or metabolites are absorbed.

Orally ingested YP is rapidly absorbed through the gastrointestinal system and approximately $70 \%$ is accumulated in the liver within 2 to 3 hours. It accumulates to a lesser extent in the heart (12\%), kidneys (4\%), pancreas (0.4\%), and brain (0.39\%), and also leads to damage in those organs [1].

It is a protoplasmic poison [1]. In liver YP damages the endoplasmic reticulum where protein synthesis occurs. It therefore, impairs the synthesis of protein including enzymes, which results in intoxication of major organs, causing irreversible anoxia of tissues which eventually leads to death. It also affects the carbohydrate and fat metabolism. There takes place competitive substitution of phosphates in these reactions, which leads to pathological changes in metabolism.

The patient with YP intoxication passes through three stages [9]:

Stage 1: Gastrointestinal symptoms and Shock- Features generally begin within minutes of ingestion, but may be delayed, and include nausea, vomiting, diarrhoea, pain abdomen. If substantial amount of yellow phosphorus is taken, CNS and cardiovascular toxicity may develop at this stage.

Stage 2: Quiescent period- It is an asymptomatic period and the patient seems to be improving. The patient may be discharged prematurely. This stage can last for 24-72 hours.

Stage 3: Nausea, protracted vomiting, diarrhoea and massive hematemesis may occur. This stage is marked by clinical deterioration mainly hepatic failure, metabolic complications, CNS toxicity, and cardiovascular toxicity. Mortality is high in this stage. Death occurs usually in 4-8 days.

There is no diagnostic test for YP poisoning. A garlic odour from breath and excreta are highly suggestive of phosphorus poisoning. Luminescent vomitus and faeces are essentially diagnostic of phosphorus. The reported mortality after YP intoxication varies from $23 \%$ to $73 \%$, and it is directly related with the ingested dose [10].

Yellow phosphorus poisoning is not an uncommon poisoning in India but toxicity to myocardium and ECG changes are reported rarely. 
YP was the most ingested poison of all the commonly available rodenticides compounds [3,11].

In a study by Nalabothu $\mathrm{M}$ et al. $44.3 \%$ of the patients ingested Phosphorous compounds [3].

Mathews et al. reported 62 patients of rodenticide poisoning during the 3 year period [11]. Most common rodenticide was yellow phosphorous (paste form) in 33 patients (53\%). In their study complications developed in 11 cases of yellow phosphorous ingestion.

Chikkaveeraiah KS et al. [4] (noted that the systems affected in their study due to YP were gastrointestinal tract (100\%), Liver (66.70\%), CVS, Nervous and respiratory systems along with associated metabolic abnormalities (66.7\%). Liver toxicity in form of acute hepatitis with or without fulminant hepatic failure is the most common form of presentation [12]. Certain rare presentations like acute hepatic cholestasis can also be seen in YP poisoning [13]. Neutropenia, Brain injury as manifested by convulsions, delirium coma and anuric renal failure are some of the other complications. Renal failure occurs because of shock and the toxic effects of phosphorus products and accumulating bilirubin on renal tubules.

Alterations in electrocardiogram readings, tachycardia, arrhythmias, atrial fibrillation, and decreased ventricular contractility have been observed in humans orally exposed to white phosphorus [14]. Ventricular tachycardia and ventricular fibrillation has also been described in YP poisoning.

Cardiovascular toxicity in YP simulating acute myocardial infarction [15] and persistent left ventricular dysfunction has been reported [16]. Acute pulmonary oedema has been reported with YP intoxication [17]. In a cross sectional study where 85 patients were studied; dysrhythmias were noted in only two patients, another four showed tachy-bradycardia [18].

Tachycardia, arrhythmia, myocardial infarction and cardiac arrest is suggestive of direct action of the phosphorus on myocardium and conduction fibres within the heart. The cardiac toxicity can also occur due to secondary peripheral vascular collapse that causes a decrease in the coronary blood flow resulting in severe myocardial ischemia $[4,18]$. Depression of the serum calcium level with an elevation in the serum phosphorus level (reversed calcium-phosphorus ratio) with electrocardiographic changes including prolongation of the QT segment, ST-segment depression, T-wave changes, and bradycardia also have been observed. YP toxicity is also secondary due to the release of phosphine gas [19].

The autopsy of patients dying from YP poisoning nearly always revealed severe damage to one or more of those four systems i.e. liver, kidney, brain, and/or cardiovascular system. The most common pathological findings in deaths have been fatty degeneration of the liver and kidneys. Fatty infiltration, necrosis, cross striations, and interstitial oedema that have been observed in the hearts of affected individuals. These alterations may be due to a direct effect on the myocardium and conduction fibres within the heart.

Severe poisoning may also manifest as severe electrolyte abnormalities e.g., hypocalcaemia, hyperchloremic hypocalcaemia, hypokalaemia and either hyperphosphatemia or hypophosphatemia [18].

Our patient presented with gastrointestinal symptoms, her symptoms abated by symptomatic treatment and she felt good. She was thus discharged in asymptomatic stage (Stage 2) but then readmitted in the third stage of YP poisoning i.e. with cardiovascular collapse. In such cases death is inevitable.

The cardiac involvement in our patient occurred 58 hours after ingestion, thus emphasizing the need of closely monitoring these patients even if asymptomatic. Hypocalcaemia was also noted in our patient also (Serum Calcium- $7.0 \mathrm{mEq} / \mathrm{L}$ ) which may have added to the toxic effect of YP poison on heart. The sudden death due to ventricular arrhythmia in our patient may have been induced by the direct toxic effect of the poison to the myocardium as demonstrated by diffuse changes in ECG (ST sagging) or peripheral circulatory failure causing myocardial ischemia. The terminal event before the death was ventricular fibrillation.

\section{Conclusion}

There is no specific antidote for yellow phosphorous. A general symptomatic treatment received during the initial phase of intoxication could invariably turn harmful for the patient as it suddenly gives impression of improvement. Early improvement should not be interpreted as meaning that serious exposure has not occurred. Ventricular arrhythmia is an unusual complication of YP poisoning which can occur late in YP poisoning. Patients with phosphorus exposure should be monitored during the first 48-72 hours after exposure with frequent laboratory checks and ECG. Patients that remain asymptomatic after this monitoring period can be discharged.

The history of poisoning should be elicited in all cases presenting with gastrointestinal symptoms.

The consumption of rat poison (Ratol) has raised an alarm over the sale of the pesticide in the open market in India. Unfortunately, till now no concrete steps have been initiated by the government to control the sale of the poison which is "silently" killing youngsters.

\section{References}

1. Brent J, Wallace KL, Burkhart KK, Phillips SD, Donovan JW (2005) Phosphorus In: Critical Care Toxicology - Diagnosis and Management of the Critically Poisoned Patient. Philadelphia, PA: Elsevier Mosby, USA.

2. Karanth S, Nayyar V (2003) Rodenticide-induced Hepatotoxicity. J Assoc Physicians India 51: 816-7. 
3. Nalabothu M, Monigari N, Acharya R (2015) Clinical Profile and Outcomes of Rodenticide Poisoning in Tertiary Care Hospital. IJSRP 5: 1-12.

4. Chikkaveeraiah SK, Marijayanth M, Reddy PK, Kaluvakuri S (2016) Clinical profile and outcome of rodenticide poisoning in patients admitted to a tertiary care teaching hospital in Mysore, Karnataka. India. Int J Res Med Sci 4: 5023-7.

5. González-Andrade F, López-Pulles R (2011) White phosphorous poisoning by oral ingestion of fire crackers or little devils: Current experience in Ecuador. Clin Toxicol 49: 29-33.

6. Ghoshal AK, Porta EA, Hartroft WS (1969) The role of lipo-peroxidation in the pathogenesis of fatty livers induced by phosphorus poisoning in rats. Am J Pathol; 54: 275-91

7. Agency for Toxic Substances and Disease Registry (ATSDR) (1997) US Department of Health and Human Services, Public Health Service. Toxicological profile for white phosphorus, USA.

8. US National Library of Medicine HSDB (2005) Phosphorus Elemental, USA.

9. Currance PL, Clements B, Bronstein AC (2005) Emergency Care for Hazardous Materials Exposure (3 ${ }^{\text {rd }}$ Edn), Elsevier Mosby, St. Louis, MO, USA.

10. McCarron MM, Gaddis GP, Trotter AT (1981) Acute yellow phosphorus poisoning from pesticide pastes. Clin Toxicol 18: 693-711.

11. Mathews M, Sreedhar S, Valliyot B, Balakrishnan (2016) Rodenticidal Poisoning in a Tertiary Centre in North Kerala. IJSR 5: 464-6.

12. Muskar A, Mehta K, Nagotkar L, Shanbag P (2011) Acute hepatic failure due to yellow phosphorus ingestion. Indian J Pharmacol 43: 355-6.

13. Lakshmi CP, Goel A, Basu D (2014) Cholestatic presentation of yellow phosphorus poisoning. J Pharmacol Pharmacother 5: 67-9.

14. Talley RC, Linhart JW, Trevino AJ, Moore L, Beller BM (1972) Acute elemental phosphorus poisoning in man: Cardiovascular toxicity. Am Heart J 84: 139-40.

15. Pietras RJ, Stavrakos C, Gunnar RM, Tobin JR Jr (1968) Phosphorus poisoning simulating acute myocardial infarction. Arch Intern Med 122: 430-4.

16. Pooja PS, Padmakumar AV, Suresh CG (2015) Persistent Left Ventricular Dysfunction in a Teenager with Yellow Phosphorous Poisoning. JCR 5: 137-40.

17. George P (2010) An Unusual Cause of Pulmonary Oedema and Its Successful Management: A Case Of Phosphorus Poisoning. J Clin Diag Res 4: $3554-7$.

18. Diaz-Rivera RS, Collazo PJ, Pons ER, Torregosa MV (1950) Acute phosphorus poisoning in man: A study of 56 cases. Medicine $29: 269-98$.

19. Sundragiri S, Tandur S (2016) Electrocardiographic profile of Agrochemical poison. IJCMR 3: 3539-42.

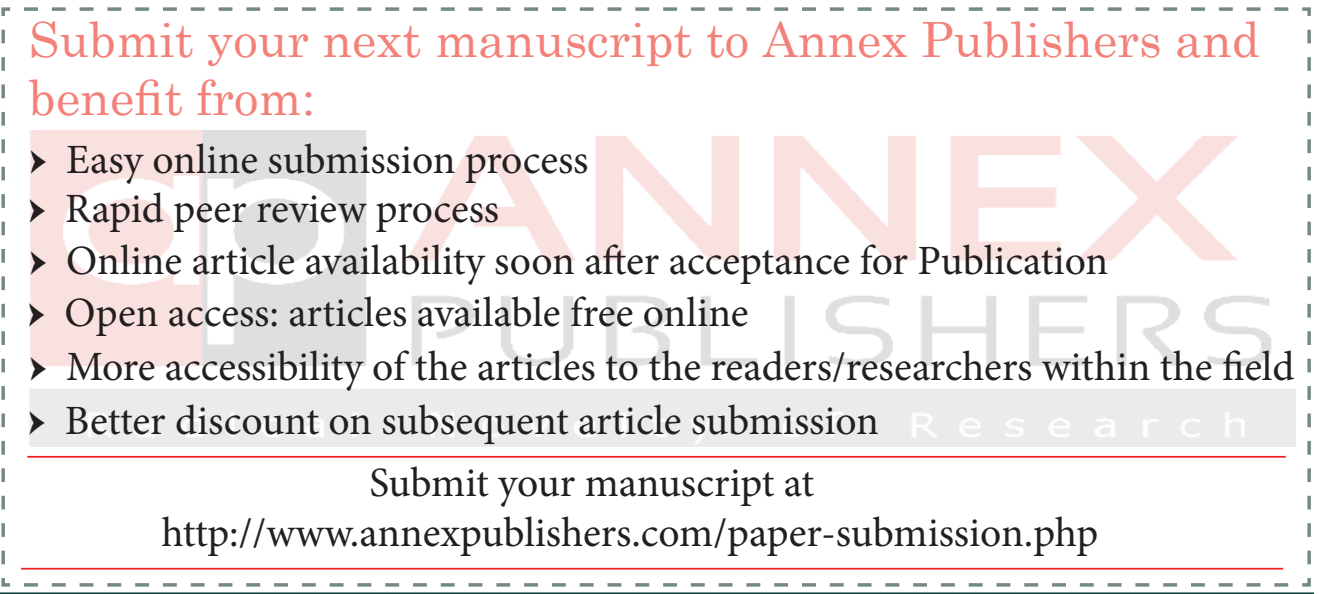

